\title{
The long Galactic bar as seen by UKIDSS Galactic plane survey
}

\author{
A. Cabrera-Lavers ${ }^{1,2}$, C. González-Fernández ${ }^{1}$, F. Garzón ${ }^{1,3}$, P. L. Hammersley ${ }^{1}$, and M. López-Corredoira ${ }^{1}$ \\ 1 Instituto de Astrofísica de Canarias, 38205 La Laguna, Tenerife, Spain \\ 2 GTC Project Office, 38205 La Laguna, Tenerife, Spain \\ e-mail: antonio.cabrera@gtc.iac.es \\ 3 Departamento de Astrofísica, Universidad de La Laguna, 8205 La Laguna, Tenerife, Spain
}

Received 31 July 2008 / Accepted 13 September 2008

\begin{abstract}
Context. Over the past decade there have been a series of results supporting the hypothesis of the existence of a long thin bar in the Milky Way with a half-length of $4.5 \mathrm{kpc}$ and a position angle of around $45^{\circ}$. This is apparently a very different structure from the triaxial bulge of the Galaxy, which is thicker and shorter and dominates the star counts at $|l|<10^{\circ}$.

Aims. In this paper, we analyse the stellar distribution in the inner Galaxy to see if there is clear evidence for two triaxial or bar-like structures in the Milky Way.

Methods. By using the red-clump population as a tracer of Galactic structure, we determine the apparent morphology of the inner Galaxy. Deeper and higher spatial-resolution near infrared photometry from the UKIDSS Galactic plane survey allows us to use inplane data even at the innermost Galactic longitudes, a region where the source confusion is a dominant effect that makes it impossible to use other databases, such as 2MASS or TCS-CAIN.

Results. We show that results previously obtained with the red-clump giants are confirmed with the in-plane data from UKIDSS GPS. There are two different structures coexisting in the inner Galactic plane: one with a position angle of $23^{\circ} .60 \pm 2^{\circ} .19$ that can be traced from the Galactic centre up to $\sim 10^{\circ}$ (the Galactic bulge), and other with a larger position angle of $42^{\circ} .44 \pm 2{ }^{\circ} .14$, that ends around $l=28^{\circ}$ (the long Galactic bar).
\end{abstract}

Key words. Galaxy: general - Galaxy: stellar content - Galaxy: structure - infrared: stars

\section{Introduction}

The nature of the morphology of the inner Galaxy is being continously unveiled as new and precise data on its stellar content is accumulated over the years. There is now little doubt, if any, that there is a stellar bar in the Milky Way. From the pioneering work in this topic of de Vaucouleurs (1964), using gas-velocity data, many insights in this direction have been produced to date using different methods, from the analysis of infrared surface brightness maps (e.g. Blitz \& Spergel 1991; Dwek et al. 1995) to studies of the asymmetry in the number counts (Weinberg 1992; Hammersley et al. 1994; Stanek et al. 1994; López-Corredoira et al. 2001). They both show far more stars at positive galactic longitudes than negative longitudes for $l<30^{\circ}$, close to the Galactic plane.

However, the exact morphology of the inner Galaxy is still the subject of some controversy, and it is being resolved as the precise stellar distribution is delineated by a succession of progessively larger scales, deeper sensitivity, and higher spatial-resolution near infrared (NIR) surveys (TMGS, Garzón et al. 1993; DENIS, Epchtein et al. 1997; 2MASS, Skrutskie et al. 2006; TCS-CAIN, Cabrera-Lavers et al. 2006; GLIMPSE, Benjamin et al. 2003; UKIDSS, Lawrence et al. 2007).

While some authors have referred to the bar as a thick structure, around $2.5 \mathrm{kpc}$ in length with a position angle of $15-30 \mathrm{de}-$ grees with respect to the Sun-Galactic centre direction (Dwek et al. 1995; Nikolaev \& Weinberg 1997; Stanek et al. 1997; Binney et al. 1997; Freudenreich 1998; López-Corredoira et al. 1999; Bissantz \& Gerhard 2002; Babusiaux \& Gilmore 2005), other researchers suggest that there is a long bar with a half length of $4 \mathrm{kpc}$ and a position angle of around 45 degrees (Weinberg 1992; Hammersley et al. 1994, 2000; Sevenster et al. 1999; Van Loon et al. 2003; Picaud et al. 2003; Benjamin et al. 2005; López-Corredoira et al. 2007).

It should be noted, however, that the references supporting the shorter bar all examine the region at $|l|<12^{\circ}$ and normally off the plane, partly due to the interstellar extinction, whereas those mentioning the long bar with the larger angle are trying to explain counts for $10^{\circ}<|l|<30^{\circ}$. This suggests that there are probably two different Galactic components coexisting in the inner Galaxy, instead of a single component alone with such a complex geometry.

In this picture, the structure present in the inner Galaxy $\left(|l|<12^{\circ}\right)$ might be interpreted as a triaxial bulge with axial ratios of 1:0.5:0.4 (López-Corredoira et al. 2000, 2005) rather than a bar, and certainly not a long straight bar. Furthermore, López-Corredoira et al. (2001) shows that a triaxial bulge + disc model cannot reproduce the observed counts in the Galactic plane for $10^{\circ}<l<30^{\circ}$, something also suggested by the NIR photometry of red-clump stars (Nishiyama et al. 2005).

Along this line, Cabrera-Lavers et al. (2007a) presents a detailed analysis of the distribution of red-clump stars in the inner Galaxy by using deep NIR photometry from the TCS-CAIN survey (Cabrera-Lavers et al. 2006). They showed that there is evidence of a bar + bulge scenario in the innermost Galaxy. While the Galactic bulge dominates the counts at higher latitudes, the long bar can be traced from the in-plane data at least from $l=18^{\circ}$ up to $l=27^{\circ}$. However, to date, no evidence of the bulge component has been found in the Galactic plane 
due to incompleteness effects in the available NIR data at those coordinates.

The intention of this paper is to repeat and complete the analysis in Cabrera-Lavers et al. (2007a) to confirm or refute these conclusions, using the best available dataset on the stellar distribution of the central Galaxy in the NIR, the United Kingdom Infrared Deep Sky Survey (UKIDSS, Lawrence et al. 2007). Using the red-clump source distribution in the inner Galactic plane to trace the $3 \mathrm{D}$ morphology of the stellar population has been performed by several authors (Hammersley et al. 2000; Stanek et al. 1994; López-Corredoira et al. 2002, 2004; Babusiaux \& Gilmore 2005; Nishiyama et al. 2005, 2006b). In this paper, we pay close attention to the potential differences in the structural parameters derived from the two datasets, TCS-CAIN (Cabrera-Lavers et al. 2007a) and UKIDSS (this work), which could then be assigned to the different stellar distributions being traced by both surveys, due to their differences in sensitivity and spatial resolution. As shown in Sects. 4.2 and 5, no significant differences are found, pointing to the fact that the majority, or at least the most prominent section, of the stellar content of the inner Milky Way has been already measured. Perhaps not surprisingly, similar results on the main structural parameter of the stellar galactic bar can also be found in Hammersley et al. (1994), where they analysed data from the TMGS (Garzón et al. 1993), with a limiting magnitude of $K=10.5$, extended with data from DIRBE (Boggess et al. 1992).

\section{The data: UKIDSS GPS}

The photometric data used here is taken from the UKIDSS Galactic plane survey (GPS) Data Release 3 . The $1 \times 1$ degree fields $\left(\Delta l=\Delta b=1^{\circ}\right)$ centred on the nominal galactic coordinates were retrieved from the UKIDSS database following the optimal query recipe outlined in Lucas et al. (2007), up to complete the range $-0.5^{\circ} \leq l \leq 30^{\circ},|b| \leq 0.5^{\circ}$. By doing so we minimize the presence of false detections (due to noise) and duplicate detections in the data. Although the authors do not distinguish galaxies from stars in the SQL query, because we are examining areas on the plane near the Galactic centre, the number of these sources per square degree should be extremely low, so that any further filtering makes little difference.

Nominal limiting magnitudes (defined as $90 \%$ completeness limits) in the Vega system of UKIDSS GPS are $J=19.77$, $H=19.00$, and $K=18.05$, with uncertainties of about $0.2 \mathrm{mag}$ (Lucas et al. 2007). However, for the inner in-plane fields used here, confusion-limited magnitudes are slightly brighter, of the order of $J=18, J=17$, and $K=16$ at $l<20^{\circ}$ (and even brighter towards the Galactic centre). These values are more than 3 mag fainter than the ones reached by 2MASS (Skrutskie et al. 2006) and 1.5 mag fainter than the ones of TCS-CAIN survey (Cabrera-Lavers et al. 2006), making UKIDSS GPS the most powerful tool available to date for studying these inner Galactic structures.

\section{Deriving distances from the red-clump population}

The red-clump population of giants has been shown to be an excellent distance indicator as it has a well-defined absolute magnitude with only a little dependence on age or metallicity (Alves 2000; Grocholsky \& Sarajedini 2002; Salaris \& Girardi 2002; Pietrzyński et al. 2003) and they are by far the most

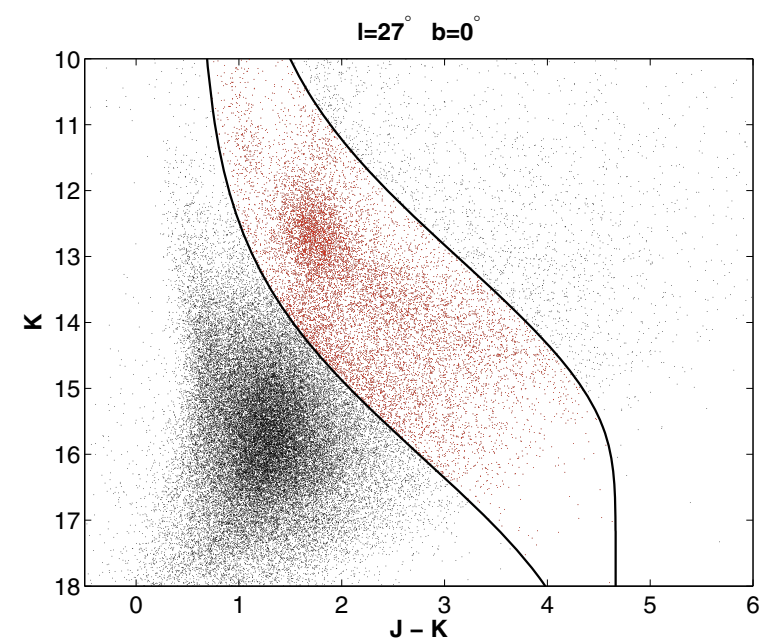

Fig. 1. Example of the use of the theoretical traces for the giant population by means of the SKY model. Stars isolated between both traces (corresponding to the KOIII and MOIII populations) are assumed to be red-clump stars. In this particular case, note the "bump" that is clearly distinguishable at $(K, J-K)=(12.8,1.8)$ that reveals the presence of an uderlying narrow structure along the line of sight.

prominent population of giants (Cohen et al. 2000; Hammersley et al. 2000), which makes them easily identifiable in a colourmagnitude diagram (CMD). There are several works where this population has been used to determine both densities and distances along different lines of sight with very interesting results. As examples, López-Corredoira et al. $(2002,2004)$ determined some parameters for the outer and inner thin discs by using this population, whilst in Cabrera-Lavers et al. $(2005,2007 \mathrm{~b})$ the thick disc was analysed in the same manner.

Cabrera-Lavers et al. (2007a, hereafter CL07) also used this red-clump method to analyse the geometry of the inner Galaxy. Briefly, in this method the red-clump stars are first isolated in the CMDs using the SKY extinction model (Wainscoat et al. 1992) to derive the theoretical traces for the giant population. These traces allow the red-clump stars to be extracted from the CMD, as they are assumed to lie between them, hence removing the contribution of dwarf stars to the counts (Fig. 1). Although this procedure includes nearly all the K-giants in the counts, the vast majority of these stars are red-clump stars. According to Flynn \& Freeman (1993), nearly 50\% of field red giants are in fact redclump stars, and this percentage even increases to $70 \%$ following López-Corredoira et al. (2002, Fig. 2). This ensures that the peak of the observed counts coincides with the peak of the red-clump distribution, hence validating the method.

The red-clump stars are then de-reddened following the relationship

$\mu=K_{\mathrm{s}}-\frac{A_{K_{\mathrm{s}}}}{A_{J}-A_{K_{\mathrm{s}}}}\left[\left(J-K_{\mathrm{s}}\right)-\left(J-K_{\mathrm{s}}\right)_{0}\right]-M_{K}$,

adopting $A_{K_{\mathrm{s}}} / E_{J-K_{\mathrm{s}}}=0.68$, from Rieke \& Lebofski (1985), and assuming $M_{K}=-1.62 \pm 0.03$ and $\left(J-K_{\mathrm{s}}\right)_{0}=0.7 \pm 0.05$ for the red-clump stars (Alves 2000; Grocholsky \& Sarajedini 2002; Bonatto et al. 2004).

Hence, we can obtain a histogram of the distance moduli along the line of sight. This distribution would reveal the presence of any single structure, as a "bump" of stars that dominates the background stars distribution (generally represented by a second-order polynomial). By fitting a Gaussian plus the background contribution the distance modulus to the structure can 
be derived, along with its apparent width (defined through the sigma of the Gaussian fit):

$N(m)=a+b m+c m^{2}+\frac{N_{\mathrm{RC}}}{\sigma_{\mathrm{RC}} \sqrt{2 \pi}} \exp \left[-\frac{\left(m-m_{\mathrm{RC}}\right)^{2}}{2 \sigma_{\mathrm{RC}}^{2}}\right]$.

The full method, originally developed by Stanek et al. (1997), as well as its uncertainties, is fully described in CL07, as well as in Nisiyahma et al. (2006a,b) and Babusiaux \& Gilmore (2005), so we refer the reader to those works for a more detailed explanation of the method.

\subsection{Completeness effects}

CL07 used NIR data from the TCS-CAIN survey (CabreraLavers et al. 2006), which provides data 1-2 mag deeper than 2MASS in the region $|l|<30^{\circ},|b|<5^{\circ}$. However, completeness limiting magnitudes were too bright to use the method for in-plane fields at $|l|<15^{\circ}$. The results obtained in their work came from a combination of in-plane data at $15^{\circ}<|l|<30^{\circ}$ and from off-plane data at $|l|<5^{\circ}$, revealing a double morphology in the inner Galaxy, a thicker structure in the innermost Galaxy (the bulge), and a larger thin structure up to $l=27^{\circ}$, constrained to the Galactic plane (the long bar).

In this work, we follow the same method as CL07 but only use the in-plane data from the UKIDSS GPS to obtain the distribution of stars in the inner Galaxy. This will confirm the previous result obtained using a combination of on- and off-plane data, as the UKIDSS GPS is notably deeper than TCS-CAIN survey in the regions of interest.

Figure 2 shows, as an example, a comparison between inplane results at $l=10^{\circ}$ coming from TCS-CAIN and UKIDSS GPS. It can be seen that the confusion-limited magnitude is brighter in the TCS-CAIN data, and this magnitude is too close to the peak of the distribution to perform a reliable fit to the data $^{1}$. The limiting magnitude is notably fainter in UKIDSS data, so in this case we can fit the distribution more accurately. In both cases, however, the distance derived from the distribution is nearly the same.

\section{Results: distribution of red-clump sources in the inner Galactic plane}

We applied the red-clump method to the UKIDSS Galactic plane survey data in steps of 1 degree for all the in-plane data at $l<30^{\circ}$. Results coming from the Gaussian, plus a second-order polynomial fit (by means of Eq. (2)), to the distance moduli distributions are shown in Table 1, as well as the distance derived to those peaks. In each case we have used a square of $0.25 \mathrm{deg}^{2}$ in the sky $\left(\Delta l=\Delta b=0.5^{\circ}\right)$ centred on the nominal galactic coordinates to extract the exact position of the peak.

Figure 3 shows the fits to the de-reddened distributions of red-clump stars for five different fields analysed in this work. In all the cases, the "bump" in the distribution is evident, and its location changes depending on the longitude of field (as the distance to the underlying structure varies). Also, a broadening in the distribution for the innermost fields respect to the field at $l=27^{\circ}$ can be noted. This is further evidence of there being a difference between $l<10^{\circ}$ and $l=27^{\circ}$, as we discuss later.

\footnotetext{
1 In CL07 a minimum difference of 0.5 mag between the limiting extinction-corrected magnitude of the field and the maximum of the Gaussian fit was used to ensure the reliability in the results. This is a conservative limit, that is also used, for example, in Babusiaux \& Gilmore (2005).
}

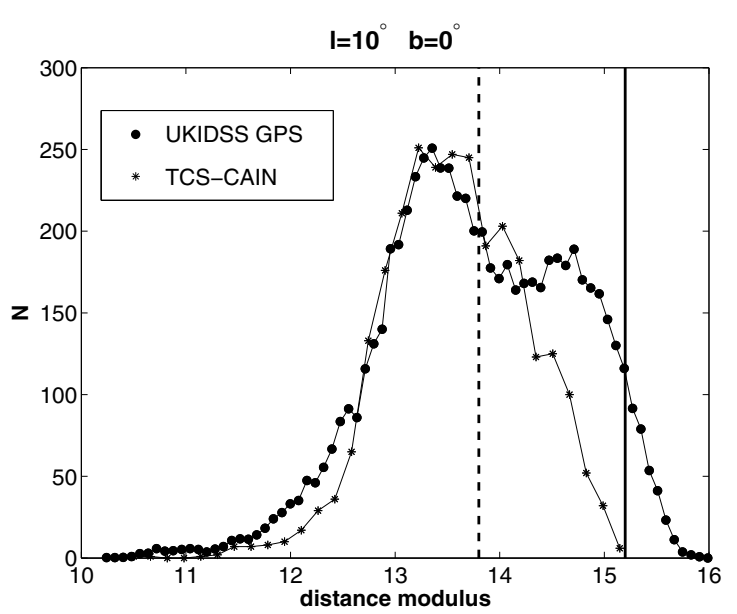

Fig. 2. Spatial distribution of red-clump stars obtained with either TCS-CAIN (asterisks), or UKIDSS GPS data (points) for the field $l=10^{\circ}, b=0^{\circ}$. Distributions have been normalized to the maxima for the comparison. Vertical lines show the limiting magnitude derived in each case. It can be seen how the completeness limit is fainter for UKIDSS data (solid line), which is sufficient to allow a fit to the data, whilst this was impossible with TCS-CAIN data, as the completeness limit was too close to the peak of the distribution (dashed line).

There are some cases where no reliable fit was obtained due to incompleteness. For the innermost fields $\left(l \leq 1^{\circ}\right)$, the limiting extinction-corrected magnitudes are too faint to reach the "bump" in the red-clump distribution, while the peak in the redclump distribution for $l=2^{\circ}$ and $l=3^{\circ}$ is too close to the limiting extinction corrected magnitude to ensure the reliability of the fit (see for example, the lower panel in Fig. 3, where the field at $l=3^{\circ}$ is shown). Therefore the results from these four fields $\left(l \leq 3^{\circ}\right)$ have been ignored for the present analysis.

There are also two fields, at $l=23^{\circ}$ and $l=24^{\circ}$, where there is no apparent "bump" in the red-clump distribution, due to an effect of extinction. At these coordinates it is well known that the extinction is very high, even in the mid-infrared (see Fig. 1 in Benjamin et al. 2005). To illustrate this, Fig. 4 shows a comparison between the two adjacent fields at $l=24^{\circ}$ and $l=25^{\circ}$. The number of stars present in both fields is clearly different, even though the sky area covered is the same. Also, the structure in the red giant branch that can be clearly noted in the $l=25^{\circ} \mathrm{CMD}$, running from $(J-K, K)=(2,13)$ to $(J-K, K)=$ $(4.5,14)$ can be also observed in the $l=24^{\circ} \mathrm{CMD}$, but appearing approximately at $(J-K, K)=(4,14)$, a value that is too close to the completeness limit of the field. Probably, a CMD that is one or two magnitudes deeper is needed to observe this structure properly, at least at those coordinates.

Figure 5 shows the red-clump distribution in the $X Y$ plane of the Galaxy obtained from the results presented in Table 1. There is a clear change in the trend of those data at $\sim l=10^{\circ}$. For the innermost fields $\left(l<10^{\circ}\right)$, the points lie very close to the expected geometry for the triaxial bulge, with a position angle respect to the Sun-Galactic centre line of $23.60 \pm 2^{\circ} .19$, in agreement with the estimates for this component (Dwek et al. 1995; López-Corredoira et al. 2005; Stanek et al. 1997; Babusiaux $\&$ Gilmore 2005). For the outer fields $\left(l>10^{\circ}\right)$, the data are well-distributed along the expected geometry for the long thin bar, deriving a position angle of $42^{\circ} .44 \pm 2.14$ for this feature (Hammersley 2000; Benjamin et al. 2005; CL07). For $l>28^{\circ}$, there is no apparent underlying structure in the red-clump population, obtaining only a continuous increase in the counts as it 
Table 1. Parameters of the Gaussian fit for the distribution of the redclump stars, and distances to the peak of these distributions for each in-plane $\left(b=0^{\circ}\right)$ field used in this work.

\begin{tabular}{ccccc}
\hline \hline$l\left(^{\circ}\right)$ & $m_{\mathrm{RC}}(\mathrm{mag})$ & $D(\mathrm{kpc})$ & $\sigma(\mathrm{mag})$ & $N_{\mathrm{RC}}$ \\
\hline 0.0 & - & - & - & - \\
1.0 & - & - & - & - \\
$2.0 *$ & $13.840 \pm 0.13$ & $5.861 \pm 0.350$ & $0.49 \pm 0.03$ & 3114.2 \\
$3.0 *$ & $13.826 \pm 0.09$ & $5.823 \pm 0.241$ & $0.50 \pm 0.08$ & 2550.9 \\
4.0 & $14.104 \pm 0.10$ & $6.619 \pm 0.304$ & $0.71 \pm 0.05$ & 4169.7 \\
5.0 & $13.996 \pm 0.12$ & $6.298 \pm 0.347$ & $0.55 \pm 0.06$ & 2750.0 \\
6.0 & $14.142 \pm 0.10$ & $6.736 \pm 0.309$ & $0.95 \pm 0.05$ & 4913.1 \\
7.0 & $13.919 \pm 0.11$ & $6.078 \pm 0.307$ & $0.80 \pm 0.06$ & 2721.4 \\
8.0 & $13.965 \pm 0.10$ & $6.208 \pm 0.285$ & $0.70 \pm 0.05$ & 2149.1 \\
9.0 & $13.925 \pm 0.08$ & $6.095 \pm 0.224$ & $0.70 \pm 0.04$ & 2001.3 \\
10.0 & $13.633 \pm 0.08$ & $5.328 \pm 0.205$ & $0.85 \pm 0.04$ & 3224.5 \\
11.0 & $14.112 \pm 0.10$ & $6.643 \pm 0.305$ & $0.50 \pm 0.05$ & 1371.0 \\
12.0 & $14.192 \pm 0.12$ & $6.892 \pm 0.380$ & $0.45 \pm 0.06$ & 1557.4 \\
13.0 & $14.027 \pm 0.10$ & $6.388 \pm 0.293$ & $0.45 \pm 0.05$ & 969.7 \\
14.0 & $14.154 \pm 0.10$ & $6.773 \pm 0.311$ & $0.40 \pm 0.05$ & 530.7 \\
15.0 & $14.114 \pm 0.11$ & $6.649 \pm 0.336$ & $0.40 \pm 0.06$ & 766.5 \\
16.0 & $14.205 \pm 0.08$ & $6.934 \pm 0.255$ & $0.40 \pm 0.04$ & 676.8 \\
17.0 & $13.458 \pm 0.08$ & $4.915 \pm 0.180$ & $0.60 \pm 0.04$ & 1320.2 \\
18.0 & $14.039 \pm 0.14$ & $6.423 \pm 0.413$ & $0.53 \pm 0.07$ & 1884.1 \\
19.0 & $13.409 \pm 0.15$ & $4.806 \pm 0.331$ & $0.69 \pm 0.08$ & 1334.1 \\
20.0 & $13.813 \pm 0.05$ & $5.789 \pm 0.133$ & $0.50 \pm 0.03$ & 1766.4 \\
21.0 & $14.024 \pm 0.12$ & $6.379 \pm 0.352$ & $0.45 \pm 0.06$ & 1163.5 \\
22.0 & $13.956 \pm 0.06$ & $6.183 \pm 0.170$ & $0.65 \pm 0.03$ & 2310.2 \\
23.0 & - & - & - & \\
24.0 & - & - & - & \\
25.0 & $13.801 \pm 0.13$ & $5.757 \pm 0.344$ & $0.35 \pm 0.06$ & 972.4 \\
26.0 & $13.786 \pm 0.10$ & $5.717 \pm 0.263$ & $0.52 \pm 0.05$ & 818.3 \\
27.0 & $13.791 \pm 0.05$ & $5.730 \pm 0.131$ & $0.45 \pm 0.02$ & 2016.9 \\
28.0 & $13.830 \pm 0.11$ & $5.834 \pm 0.295$ & $0.60 \pm 0.05$ & 1301.7 \\
\hline
\end{tabular}

* The results coming for this field are no reliable due to incompleteness.

corresponds with a typical disc field. This places the end of the long bar at approximately $l=28^{\circ}$, coincident with the previous result of CL07.

There are two points, those corresponding to $l=17^{\circ}$ and $l=19^{\circ}$ that lie far from the expected location for this longer structure that are observed near the structure with a smaller position angle instead. The higher dispersions obtained in those fields compared to the rest of the in-plane fields at $l>10^{\circ}$ (see last column in Table 1) could be evidence that these fields are significantly affected by extinction (although a reliable fit can be performed in terms of completeness-limiting magnitudes). A similar result was obtained in CL07 for $(l, b)=\left(20^{\circ},-0.5^{\circ}\right)$, and it is well known that there is a major star formation region close to those coordinates (Garay et al. 1998; Sridharan et al. 2005). The effect of the stellar ring cannot even be discarded, as the position obtained for the red-clump maxima in those fields is coincident with the expected for this component (López-Corredoira et al. 2001). In any case, the over-density associated with the long Galactic bar is apparently somewhat inhomogeneous, as previously noted by Picaud et al. (2003) at $l=20^{\circ}$ and $l=21^{\circ}$.

It has to be noted that the distances of the maxima obtained via red-clump fitting are not coincident with the distances of the density maxima along the line of sight. In CL07 (Sect. 6.2) and López-Corredoira et al. (2007, Appendix A), it is demonstrated how the position angle derived with the red-clump method differs more than the real one as the thickness of the underlying structure increases. For the Galactic bar, which is a long and thin structure, differences are close to the own uncertainties of the method, hence the position angle derived for this structure

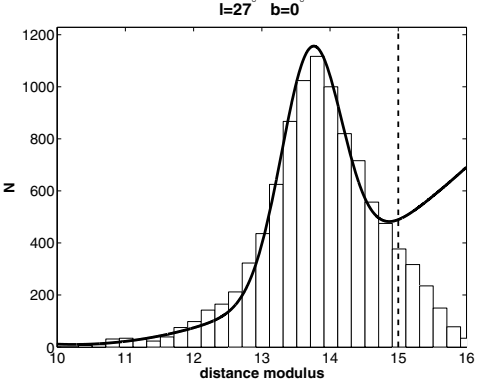

$\mathrm{l}=20^{\circ} \quad \mathrm{b}=0^{\circ}$

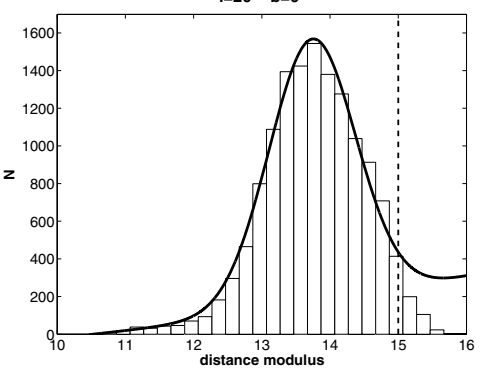

$\mathrm{l}=12^{\circ} \quad \mathrm{b}=0^{\circ}$

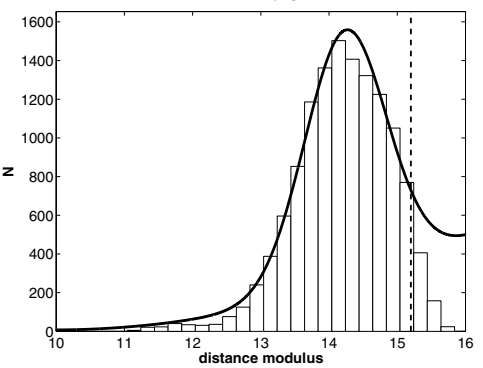

$l=6^{\circ} \quad b=0^{\circ}$

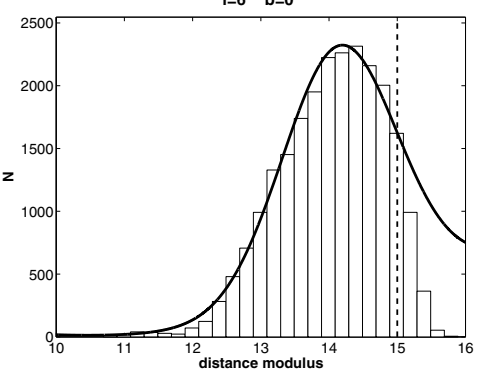

$l=3^{\circ} \quad b=0^{\circ}$

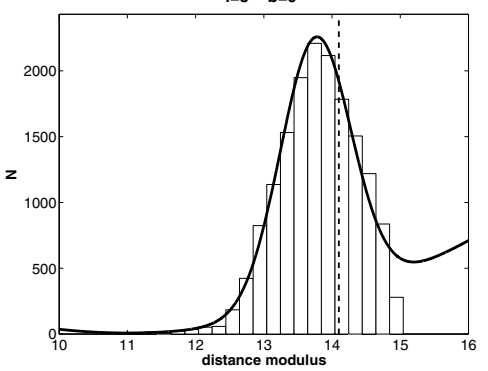

Fig. 3. Histograms of the distance modulus (using $0.2 \mathrm{mag}$ bins) in five of the fields used in this work. Fits of Eq. (2) to the histograms are shown as solid curves, while vertical dashed lines show the limiting extinction corrected magnitudes in each field.

is nearly coincident with the real one. For the triaxial bulge, the difference is higher, producing a variation of $8-10^{\circ}$ with respect to the real position angle of the bulge. Hence, the position angle for this component might be around $13-15^{\circ}$, coincident with the value previously obtained in CL07. 

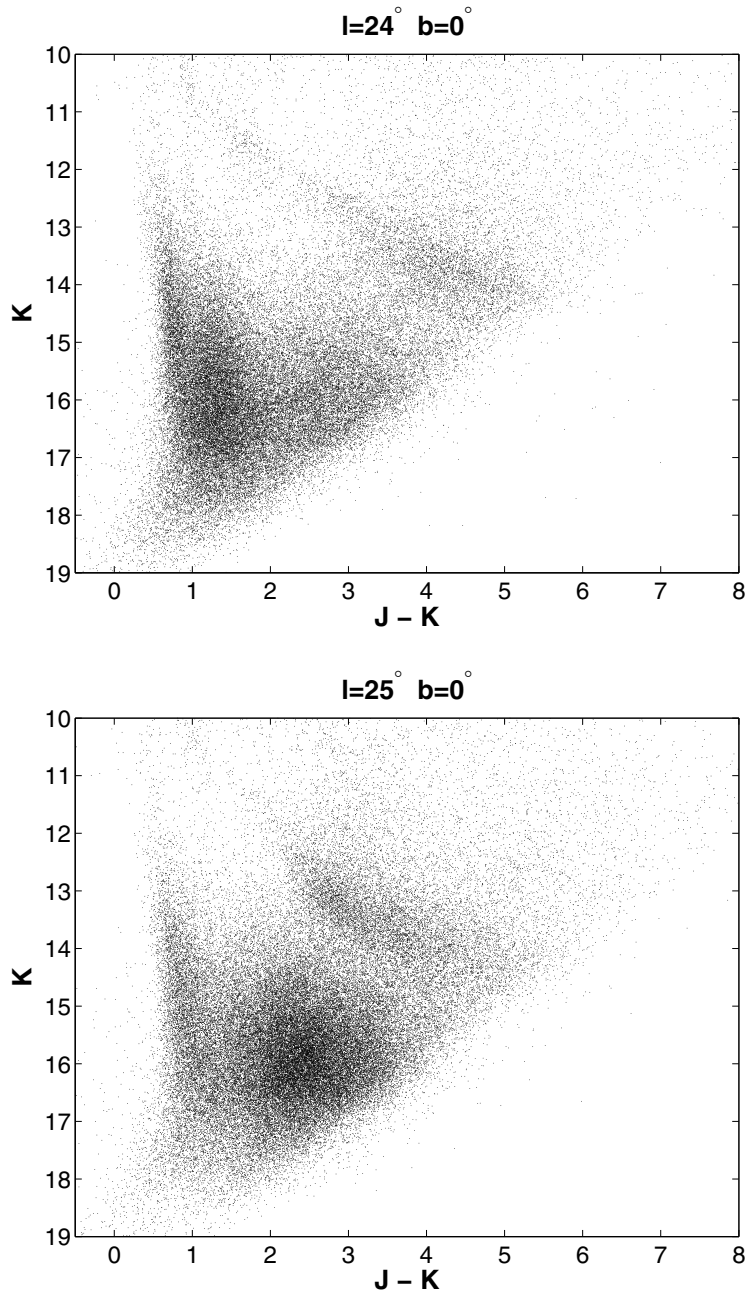

Fig. 4. CMDs corresponding to the in-plane fields $l=24^{\circ}$ (above) and $l=25^{\circ}$ (below). It can be noted how there is no apparent morphologies in the giant branch at $l=24^{\circ}$, due to the high extinction present in this region.

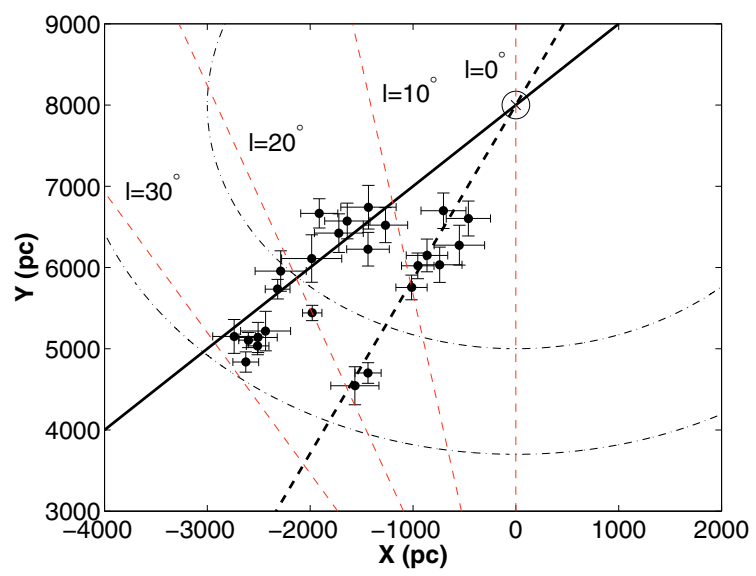

Fig. 5. Spatial distribution of red-clump giants maxima in the $X Y$-plane, with the Sun at $(0,0)$ and the Galactic centre at $(0,8000 \mathrm{pc})$ marked with a big circle. Two possible configurations for the observed distribution are also shown: a feature with a position angle of $45^{\circ}$, and another one with a position angle of $25^{\circ}$ (dashed line). Dot-dashed lines define two circles with radii $4.5 \mathrm{kpc}$ and $3 \mathrm{kpc}$, respectively, while different lines of sight towards the inner Galaxy are also shown in intervals of $10^{\circ}$ in longitude. Error bars have been estimated from the distance uncertainties, assuming these divided equally in both the $X$ - and $Y$-axes.

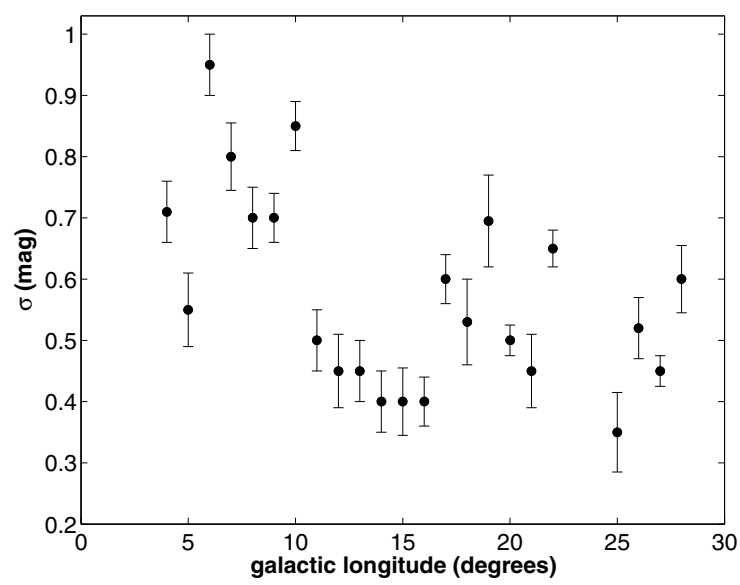

Fig. 6. Variation in the width of the red-clump distribution with the galactic longitude.

\subsection{Thickness of the components}

The dispersion in distance obtained for each field (represented by $\sigma$ in Table 1) also shows a dependence on the Galactic longitude (see Fig. 6). It is clear that, for the innermost fields $\left(l<10^{\circ}\right)$, the peak of the red-clump distribution broadens notably with respect to the one obtained for the larger Galactic longitude fields (also observed in Fig. 3). We obtain a mean value of $\langle\sigma\rangle=0.751 \mathrm{mag}$ (with a standard deviation of $0.128 \mathrm{mag}$ ) for $l \leq 10^{\circ}$, while this average is notably smaller for $l>10^{\circ}$, with $\langle\sigma\rangle=0.496 \mathrm{mag}$ (and a standard deviation of 0.098). This is indicative of the structure we are observing at $l<10^{\circ}$ being different to the one that dominates at $l>10^{\circ}$, as the width of the distributions are different by more than $2.5 \sigma$ over the average. The same effect was also noted by CL07 and López-Corredoira et al. (2007, Fig. 4). If we perform a linear fit to those points in Fig. 6 we obtain: $\sigma=0.74-0.01 l$, not very far from the $\sigma=0.64-0.004 l$ obtained in López-Corredoira et al. (2007). As expected, the triaxial bulge is notably thicker along the line of sight than the long Galactic bar, hence the width of the redclump peaks for the fields associated to this component might be higher to those of the long bar, which is assumed to have a halfwidth of about $0.6 \mathrm{kpc}$ (López-Corredoira et al. 2007). CL07 also obtained that for in-plane fields at $l>15^{\circ}$. There were very homogeneous range of dispersions in the $\sigma$ of the distribution with an average of $\sigma=0.49 \mathrm{mag}$, nearly coincident with those obtained here, which reinforces our conclusions.

There is also a notable increase in the number of red-clump sources for the innermost fields (see last column in Table 1). The number of red-clump stars at $l<10^{\circ}$ are on average nearly three times the values obtained at $l>10^{\circ}$. This indicates that, in the region where the contrast between the triaxial bulge and the long bar is higher, the long bar lies completely embedded in the bulge counts, making it impossible to note its contribution to the histogram counts (see, for example, Fig. 2 and middle panel in Fig. 3).

\subsection{Comparison with CLO7}

We show in Fig. 7 the results obtained in CL07 for the combination of in-plane data at $l>15^{\circ}$ and off-plane data for $l<10^{\circ}$, to compare with those of Fig. 5. The agreement between both sets of data is evident. While off-plane results in CL07 are coincident with the in-plane data of UKIDSS GPS in the innermost fields, 


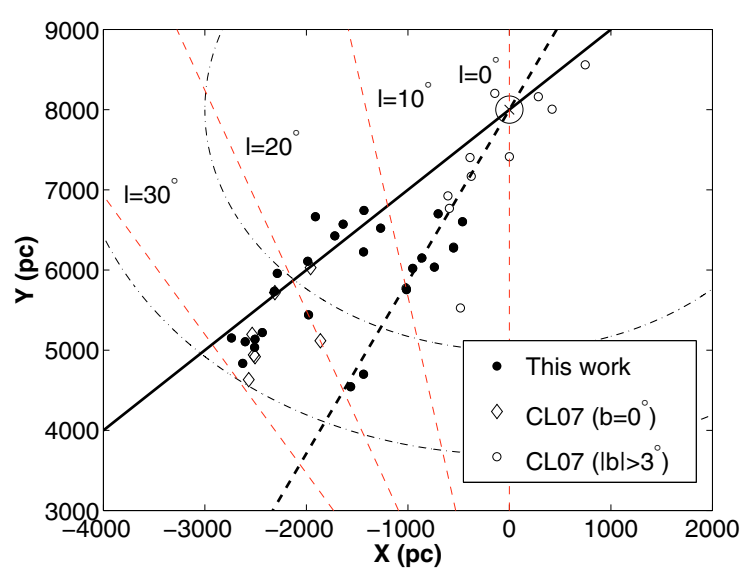

Fig. 7. Same as Fig. 5, but now including the results obtained in CL07 for a combination of in-plane and off-plane data from TCS-CAIN survey.

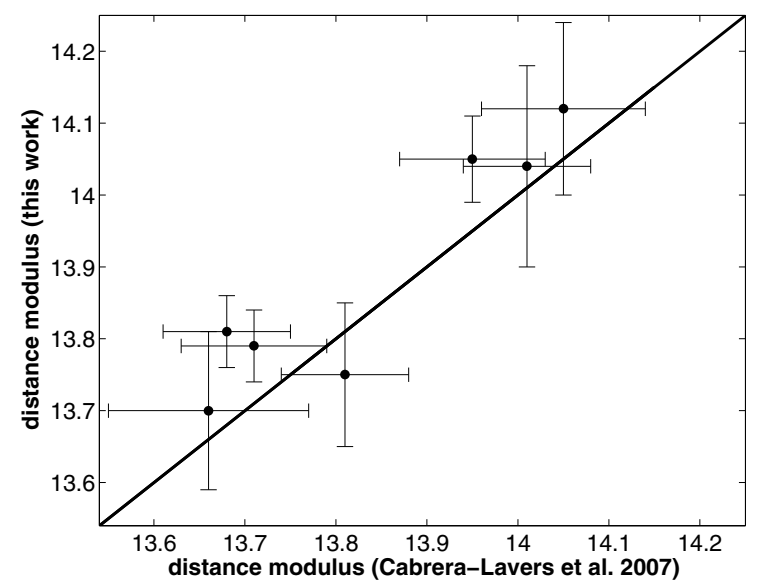

Fig. 8. Comparison between the distance modulus obtained in this work data with that obtained in CL07 for the same fields. The solid line shows the 1:1 ratio. There is an offset of 0.05 mag between both data sets related to the different characteristics of the $K$ filters used in both surveys.

data for $l>10^{\circ}$ in UKIDSS GPS coincides with the in-plane fields used in CL07.

The mean difference in distance moduli between the inplane fields in common analysed in this work and in CL07 is $\Delta \mu=0.05 \pm 0.02 \mathrm{mag}$ (see Fig. 8). By comparing the photometry of TCS-CAIN data and UKIDSS GPS in several test fields, we obtained mean differences (UKISS-TCS) of $\Delta J=0.015 \mathrm{mag}$ $\left(\sigma_{J}=0.183\right)$ and $\Delta K=0.167 \mathrm{mag}\left(\sigma_{K}=0.256\right)$. The larger difference in the $K$ data is probably due to the different characteristics of the $K$ filter used in both surveys. In any case, a difference in distance modulus of $0.05 \mathrm{mag}$ yields to a difference in distance of only $150 \mathrm{pc}$ on average, close to the distance uncertainties for each individual field. Hence, the results coming from CL07, and this work can be considered equivalents in terms of photometry, and are comparable.

\subsection{Systematic errors in the absolute magnitude of the red-clump population}

A recent recalculation of the absolute magnitude for red-clump stars by Groenewegen (2008) gives a value slightly different to what is assumed in this work $\left(M_{K}=-1.62 \pm 0.03\right)$. It was found that the absolute magnitude determination for red-clump stars

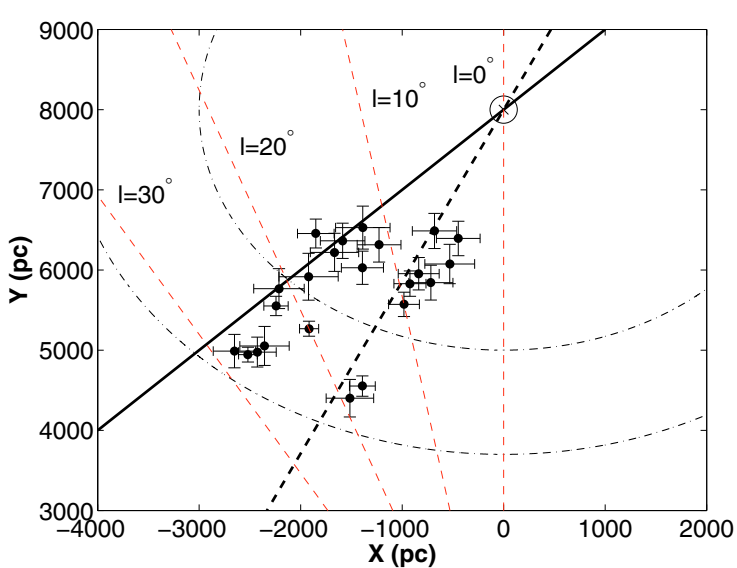

Fig. 9. Same as Fig. 5, but assuming an absolute magnitude of $M_{K}=$ -1.54 for the red-clump stars.

via Hipparcos data was biased to brighter stars, and the real value was slightly fainter: $M_{K}=-1.54 \pm 0.04$. This adds a systematic uncertainty of $0.08 \mathrm{mag}$ in the values obtained in this work, which translates into differences up to $0.15 \mathrm{kpc}$ for the closest points $(D=5 \mathrm{kpc})$ and $0.20 \mathrm{kpc}$ for the farthest ones $(D=6.5-7 \mathrm{kpc})$. This possible effect, however, does not affect the main conclusions of the work presented here, as the geometry observed in the inner Galaxy remains the same (Fig. 9). The measured points move closer by nearly the same distance along the line of sight, and the two observed structures can be traced unambiguosly. Values for the position angles are also slightly different, as the distance to the Galactic centre has been kept at $8 \mathrm{kpc}: 39.97 \pm 2.22$ for the long bar and $20^{\circ} .93 \pm 2.61$ for the triaxial bulge. However, they are still clearly different and the two-component geometry is evident.

\section{Conclusion}

By using the deepest wide area NIR data available to date for analysing the inner Galactic plane, we find that there are two different structures coexisting in the inner $4 \mathrm{kpc}$ of the Milky Way. From the Galactic centre to $l=10^{\circ}$, the distribution of red-clump sources traces a thicker structure with a position angle with respect to the Sun-Galactic centre line of $23^{\circ} .60 \pm 2^{\circ} .19$, while for $l \leq 10^{\circ}$ the red-clump sources follow a more inclined structure that can be traced up to $l=28^{\circ}$ with a position angle of $42^{\circ} .44 \pm 2.14$, which ends around $l=28^{\circ}$.

This result corroborates the previous hypothesis of Hammersley et al. (2000) that the Milky Way presents a inner triaxial bulge, together with a long thinner structure, which appears as a long Galactic bar, with a semi-length of about $4.5 \mathrm{kpc}$ from the Galactic centre. There is also evidence that the inner triaxial bulge has a "boxy" morphology (López-Corredoira et al. 2000, 2005), but nothing in that sense can be said from the analysis here.

Previous works in the same direction (CL07 and López-Corredoira et al. 2007) could not analyse the inner Galactic plane due to the shallowness in their NIR data. For this reason, they had to use a combination of on and off plane data to reach the same conclusions as ours. Some other researchers have also pointed to this double morphology in the Milky Way and suggested that the inner Galaxy is dominated at higher latitudes by the Galactic bulge, while the star counts in the plane are dominated by the long bar (Sevenster et al. 1999). The result 
presented here confirms that the same picture can be observed close to the Galactic plane.

This suggested scenario of a triaxial bulge + long bar for the Galaxy is plausible since many galaxies have triaxial bulges contained within a primary stellar bar (Friedli et al. 1996), and it is supported by recent $N$-body simulations about secular galactic evolution $^{2}$ (Athanassoula 2005; Athanassoula \& Beaton 2006) and also by NIR observations in external galaxies (Beaton et al. 2005). Boxy bulges are in fact a part of the long bar (Athanassoula 2005), and this is what is seen in the Milky Way.

Acknowledgements. We gratefully acknowledge the anonymous referee for very interesting suggestions that have notably improved the quality of this paper. This work is based on data from the 3rd data release of the UKIRT Infrared Deep Sky Survey, UKIDSS (www. ukidss.org), which is described in detail in Lawrence et al. (2007) and Lucas et al. (2007).

\section{References}

Alves, D. R. 2000, ApJ, 539, 732

Athanassoula, E. 2005, MNRAS, 358, 1477

Athanassoula, E., \& Beaton, R. L. 2006, MNRAS, 693

Babusiaux, C., \& Gilmore, G. 2005, MNRAS, 358, 1309

Beaton, R. L., Athanassoula, E., Majewski, S. R., et al. 2005, BAAS, 37, 1387

Benjamin, R. A., Churchwell, E., Babler, B. L., et al. 2003, PASP, 115, 953

Benjamin, R. A., Churchwell, E., Babler, B. L., et al. 2005, ApJ, 630, L149

Blitz, L., \& Spergel, D. N. 1991, ApJ, 379, 631

Binney, J., Gerhard, O. E., \& Spergel, D. 1997, MNRAS, 288, 365

Bissantz, N., \& Gerhard, O. E. 2002, MNRAS, 330, 591

Boggess, N. W., Mather, J. C., Weiss, R., et al. 1992, ApJ, 397, 420

Bonatto, C., Bica, E., \& Girardi, L. 2004, A\&A, 415, 571

Cabrera-Lavers, A., Garzón, F., \& Hammersley, P. L. 2005, A\&A, 433, 173

Cabrera-Lavers, A., Garzón, F., Hammersley, P. L., Vicente, B., \& González-Fernández, C. 2006, A\&A, 453, 371

Cabrera-Lavers, A., Hammersley, P. L., González-Fernández, C., et al. 2007a, A\&A, 465, 825

Cabrera-Lavers, A., Bilir, S., Ak, S., Yaz, E., \& López-Corredoira, M. 2007b, A\&A, 464, 565

Cohen, M., Hammersley, P. L., \& Egan, M. P. 2000, AJ, 120, 3362

de Vaucouleurs, G. 1964, in The Galaxy and the Magellanic Clouds Interpretation of velocity distribution of the inner regions of the Galaxy, IAU Symp., 20, 195

Dwek, E., Arendt, R. G., Hauser, M. G., et al. 1995, ApJ, 445, 716

Epchtein, N. 1997, in The Impact of Large Scale Near-IR Sky Surveys, ed. F. Garzón, N. Epchtein, A. Omont, B. Burton, \& P. Persi (Dordrecht: Kluwer), 15
Flynn, C., \& Freeman, C. 1993, A\&AS, 97, 835

Freudenreich, H. T. 1998, ApJ, 492, 495

Friedli, D., Wozniak, H., Rieke, M., Martinet, L., \& Bratschi, P. 1996, A\&AS, 118,461

Garay, G., Moran, J. M., Rodríguez, L. F., \& Reid, M. J. 1998, ApJ, 492, 635

Garzón, F., Hammersley, P. L., Mahoney, T., et al. 1993, MNRAS, 264, 773

Grocholski, A. J., \& Sarajedini, A. 2002, AJ, 123, 1603

Groenewegen, M. A. T. 2008, A\&A, 488, 935

Hammersley, P. L., Garzón, F., Mahoney, T., \& Calbet, X. 1994, MNRAS, 269, 753

Hammersley, P. L., Garzón, F., Mahoney, T. J., López-Corredoira, M., \& Torres, M. A. P. 2000, MNRAS, 317, L45

Lawrence, A., Warren, S. J., Almaini, O., et al. 2007, MNRAS, 379, 1599

López-Corredoira, M., Garzón, F., Hammersley, P. L., Mahoney, T. J., \& Calbet, X. 1997, MNRAS, 292, 15

López-Corredoira, M., Hammersley, P. L., Garzón., F., Simonneau, E., \& Mahoney, T. J. 2000, MNRAS, 313, 392

López-Corredoira, M., Hammersley, P. L., Garzón, F., et al. 2001, A\&A, 373, 139

López-Corredoira, M., Cabrera-Lavers, A., Garzón, F., \& Hammersley, P. L. 2002, A\&A, 394, 883

López-Corredoira, M., Cabrera-Lavers, A., Gerhard, O., \& Garzón, F. 2004, A\&A, 421, 953

López-Corredoira, M., Cabrera-Lavers, A., \& Gerhard, O. E. 2005, A\&A, 439, 107

López-Corredoira, M., Cabrera-Lavers, A., Mahoney, T. J., et al. 2007, AJ, 133, 154

Lucas, P. W., Hoare, M. G., Longmore, A., et al. 2007 [arXiv: 0712. 0100]

Nikolaev, S., \& Weinberg, M. D. 1997, ApJ, 487, 885

Nishiyama, S., Nagata, T., Baba, D., et al. 2005, ApJ, 621, L105

Nishiyama, S., Nagata, T., Kusakabe, N., et al. 2006a, ApJ, 638, 839

Nishiyama, S., Nagata, T., Sato, S., et al. 2006b, ApJ, 647, 1093

Picaud, S., Cabrera-Lavers, A., \& Garzón, F. 2003, A\&A, 408, 141

Pietrzyński, G., Gieren, W., \& Udalski, A. 2003, AJ, 125, 2494

Rieke, G. H., \& Lebovsky, M. J. 1985, ApJ, 288, 618

Salaris, M., \& Girardi, L. 2002, MNRAS, 337, 332

Sevenster, M., Prasenjit, S., Valls-Gabaud, D., \& Fux, R. 1999, MNRAS, 307, 584

Skrutskie, M. F., Cutri, R. M., Stiening, R., et al. 2006, AJ, 131, 1163

Sridharan, T. K., Beuther, H., Saito, M., Wyrowski, F., \& Pchilke, P. 2005, ApJ, 634, L57

Stanek, K. Z., Mateo, M., Udalski, A., et al. 1994, ApJ, 429, L73

Stanek, K. Z., Mateo, M., Udalski, A., Szymański, M., et al. 1997, ApJ, 477, 163 van Loon, J. Th., Gilmore, G. F., Omont, A., et al. 2003, MNRAS, 338, 857

Wainscoat, R. J., Cohen, M., Volk, K., Walzer, H. J., \& Schwartz, D. E. 1992 , ApJS, 83, 111

Weinberg, M. D. 1992, ApJ, 384, 81

\footnotetext{
${ }^{2}$ In fact, the ratio of sizes between the semi-major axis of the long bar and the major axis of the triaxial bulge in the simulations is 1.5 , nearly coincident what it is observed in Our Galaxy.
} 